BAwDEN, F. C. (1958). J. gen. Microbiol. 18, 751-766

\title{
Reversible Changes in Strains of Tobacco Mosaic Virus from Leguminous Plants
}

\author{
BY F. C. BAWDEN \\ Rothamsted Experimental Station, Harpenden, Hertfordshire
}

\begin{abstract}
SUMMARY: Strains of tobacco mosaic virus (TMV) obtained from systemicallyinfected leguminous plants in Nigeria and India changed their properties greatly when propagated in different hosts. From systemically-infected tobacco, they closely resemble type TMV and share many antigens with it, but from systemically-infected French bean, they differ from it by at least as much as any previously described strains. The different forms of the viruses share few antigens, have different amino acid constitutions, electrophoretic behaviour and resistance to inactivation by ultraviolet radiation, and they produce different kinds of local lesion in Nicotiana glutinosa. Isolates derived from single local lesions behave like the original bulk cultures, and as the changes are also reversible they seem unlikely to occur simply because different hosts select different strains from existing mixtures. The electrophoretic patterns, particularly of preparations from beans, show that infected plants contain more than one anomalous product; both forms of the viruses may be produced in all hosts, but there is no evidence that sap from systemically-infected beans contains the tobacco forms in amounts needed to cause infection. The change after transfer to a new host apparently occurs because mutants produced in that host are favoured over the infecting form because they move more readily and become systemic. The bean forms multiply more extensively in tobacco at above $30^{\circ}$ than at $20^{\circ}$ and above $30^{\circ}$ seem not to produce forms that give a systemic mosaic; many tobacco plants at $20^{\circ}$ develop only local lesions, and systemic infection, indicating the occurrence of the tobacco forms, happens more often in young seedlings than when mature plants are inoculated.
\end{abstract}

Some varieties of French bean (Phaseolus vulgaris L.) produce discrete necrotic local lesions when infected with tobacco mosaic virus (Price, 1930; Silberschmidt \& Kramer, 1941) and are often used to assay virus preparations. There seems to be no record of any other kind of symptom caused by tobacco mosaic virus in leguminous plants until Lister \& Thresh (1955) reported that a systemic disease of cow-pea (Vigna unguiculata (L.) Walp.) and Bengal bean (Mucuna aterrima Holland) in Nigeria was caused by a strain of this virus, which also caused systemic symptoms in the fourteen varieties of French bean they inoculated. Bawden (1956) confirmed that this disease is caused by a strain of tobacco mosaic virus and reported that, as obtained from systemically-infected beans, this strain differs greatly in its constitution and properties from when obtained from systemically-infected tobacco plants. He also reported that southern sann-hemp mosaic in India (Capoor, 1950) is caused by a strain of tobacco mosaic virus, which also undergoes comparable changes when transferred from leguminous to solanaceous plants. The present paper describes some of the unusual behaviour of these virus strains. 


\section{METHODS}

Most of the work was done with a virus culture from cowpea kindly supplied by $\mathrm{Mr}$ R. M. Lister from Nigeria; this will be called the cowpea virus and, for convenience, as obtained from systemically-infected leguminous plants, it will be referred to as the bean form and from systemically-infected tobacco plants as the tobacco form. The two forms of the southern sann-hemp virus, which was kindly supplied by Dr S. P. Capoor, will be similarly distinguished.

The French bean variety mostly used was Prince, which gives neither local nor systemic symptoms with the type strain of tobacco mosaic virus, but Pinto beans, which give necrotic local lesions and no systemic symptoms with the type strain, were sometimes inoculated. Inoculations were usually made to the first-formed true leaves, at the time the first trifoliate one was unfolding. The tobacco (Nicotiana tabacum L.) was Judy's Pride, a variety of the White Burley type. Local lesion assays with both forms of the two viruses were made on $N$. glutinosa. Unless otherwise specified, all plants were raised and kept in a glasshouse heated during winter, with no supplementary lighting and with temperature fluctuating around a mean of $20^{\circ}$. Except when doing assays, leaves to be inoculated were usually lightly dusted with 'Celite', a diatomaceous earth that facilitates transmission, before they were rubbed over their upper surfaces with the fore-finger wetted with inoculum (usually undiluted sap) and then rinsed in a stream of water.

Antisera were prepared by injecting rabbits intravenously with purified virus preparations. Precipitation tests were usually made with antiserum at a constant dilution; $1 \mathrm{ml}$. of suitably diluted antiserum was added to each of a series of tubes containing $1 \mathrm{ml}$. antigen solution at different concentrations. The tubes were placed in a glass-fronted water bath at $50^{\circ}$, with the fluid columns half immersed to ensure continuous mixing, and illuminated from behind so that the formation of precipitates could be continuously observed.

Purified virus preparations were made by precipitating the virus from clarified sap successively with ammonium sulphate and acid. The sap was usually clarified by heating to $58^{\circ}$ and low-speed centrifugation, and after precipitation with salt and acid the partially purified preparations were incubated overnight at $36^{\circ}$ with $0.1 \mathrm{M}$-sodium citrate at $\mathrm{pH} 7$. They were then clarified, the virus precipitated again with ammonium sulphate and acid, resuspended at $\mathrm{pH} 7$, sedimented by ultracentrifugation, taken up in and dialysed against distilled water. Preparations of the bean form were less easy to purify than were preparations of the tobacco form, and the bean form also went into solution less readily after precipitation with salt or acid. Salt-free preparations of both forms remained clear indefinitely and when more concentrated than about $20 \mathrm{~g}$./1. they usually produced a liquid crystalline layer when left undisturbed. Originally clear solutions of the bean form when kept in $0.1 \mathrm{M}$ phosphate buffer ( $\mathrm{pH} 7$ ) became milky within a few days at $18^{\circ}$ and more slowly at $0^{\circ}$. Left undisturbed in this medium the virus settled out as a white fibrous precipitate, which in some ways resembles that produced from preparations of the type strain of tobacco mosaic virus with nicotine and salt 
(Bawden \& Pirie, 1940). A difference, though, is that vigorous shaking does not disperse the fibres of the bean form to give a clear fluid. Preparations of the tobacco form, like those of other strains of tobacco mosaic virus, do not become insoluble in phosphate buffer. In the insoluble state produced by phosphate, the bean form does not move in an electric field at $\mathrm{pH} 7$.

Electrophoretic measurements were made in a Perkin-Elmer apparatus, model $38 \mathrm{~A}$, with the Philpot-Svenson optical system, and a potential gradient of $9 \cdot 5 \mathrm{~V} . / \mathrm{cm}$.

\section{RESULTS}

Symptoms in bean, tobacco and Nicotiana glutinosa

The symptoms produced by the cowpea virus in Prince beans vary with time of the year, but Pl. 1, figs. 1 and 2 , give an idea of the most usual effects. Plate 1, fig. 1 is a photograph of a first-formed leaf that was inoculated from systemically-infected beans and shows the many local lesions that often develop; these are white or yellow, superficially necrotic, and give the appearance of etched rings or patterns. Such local lesions do not occur when beans are inoculated with the tobacco form of the virus, and the inoculated leaves remain symptomless. Systemic symptoms are similar whether the inoculum comes from systemically-infected bean or tobacco plants, but many plants inoculated from tobacco fail to show symptoms and those that do take 5-7 days longer to do so than plants inoculated from beans. The first systemic symptoms are usually a clearing of the veins of the young trifoliate leaves, which may also then become bronzed and wilt slightly. Later-formed leaves show a mosaic of light and dark green areas, and as the length of infection increases so the newly produced leaves show increasingly obvious symptoms; in addition to mottling, they may show large blisters and other deformities, the laminae may be so decreased that little remains except the main veins; many leaves also produce enations, abnormal outgrowths from the main or subsidiary veins (Pl. 1, fig. 2).

When inoculated with the bean form, Pinto beans behave like Prince beans and give similar systemic symptoms, but when inoculated with the tobacco form they give dark necrotic local lesions, resembling those caused by the type strain of tobacco mosaic virus (TMV); these are the only symptoms and the uninoculated leaves remain normal in appearance and free from virus.

When first obtained from India the sann-hemp virus produced a milder disease in Prince beans than that caused by the cowpea virus, but after propagation for 2 years in the glasshouses at Rothamsted it has increased in virulence and both viruses now cause similar symptoms. This is unlikely to be because of contamination with the cowpea virus, for whereas the cowpea virus has also changed in that it has become increasingly difficult to transfer it from bean and get systemic infection in tobacco, the bean form of the sannhemp virus still produces systemic infection in tobacco as readily as at first.

Both the cowpea and sann-hemp viruses cause systemic symptoms in tobacco that closely resemble those caused by type TMV, a mottling in various shades of green, with occasional yellow or necrotic spots; the growth of the 
plant and size of leaf is decreased and the leaves are also often somewhat deformed. The only way of diagnosing whether infection is by type TMV or the tobacco forms of the cowpea or sann-hemp viruses is to inoculate bean plants and see whether they become systemically infected. Tobacco leaves inoculated with the tobacco forms of these viruses sometimes, as with type TMV, produce faint chlorotic spots, but usually they show no local lesions. Tobacco leaves inoculated with the bean form usually produce local lesions, but their distinctness and type vary; sometimes they are brown necrotic spots, sometimes chlorotic with minute necrotic centres. Many plants produce lesions only on the inoculated leaves, but others develop systemic symptoms of the mosaic type, which also take 5-7 days longer to appear than in plants inoculated with the tobacco forms, even when the inocula of the tobacco forms are diluted almost to the infection end-point.

Whether the bean form of the cowpea virus produces a systemic infection depends greatly on the age and condition of the tobacco plants when they are inoculated. The chances of it doing so decrease as the age of the inoculated plant increases and as its rate of growth decreases; they are greatest with young rapidly growing plants with 3-5 leaves 2-3 in. long. When the virus was first obtained from Nigeria, undiluted sap from systemically-infected beans, inoculated to such plants with the addition of 'Celite', always infected all of them systemically, and it infected about half of those which were older and had leaves 6-10 in. long. Now systemic infection is more difficult to get; it rarely occurs in the older type of plant and more than half of inoculated young seedlings also often show local lesions only. The tobacco form gives systemic infections, apparently with equal readiness, both in these kinds of tobacco plants and in ones much older and growing more slowly.

The symptoms caused by the bean forms of the cowpea and sann-hemp viruses in tobacco depend on the temperature at which the inoculated plants are kept. Those symptoms already described relate to ordinary glasshouse conditions with a mean temperature of $20^{\circ}$. At constant temperatures of $30-35^{\circ}$ there are no symptoms, either local or systemic; despite this the virus multiplies and reaches larger amounts in such symptomless inoculated leaves than when the plants are kept at lower temperatures and show lesions. When, after a week or so at over $30^{\circ}$, the plants are put at ordinary glasshouse conditions, the inoculated leaves develop large necrotic rings or spots. When kept for longer at $30^{\circ}$, occasional necrotic spots may also occur on uninoculated leaves after the plants are returned to ordinary glasshouse conditions, but there is no general systemic infection; such plants rarely produced mosaic symptoms when maintained in the glasshouse for long periods, although a few did after 2 weeks or more. It seems that, at the higher temperatures, the bean form multiplies as such in inoculated tobacco leaves and occasionally moves from these, but it becomes established in only a few isolated areas where it again multiplies without producing any of the tobacco form. The tobacco form, although it may not reach such high concentrations in tobacco plants kept at $36^{\circ}$ as at lower temperatures, readily multiplies in them, systemically invades them, and causes mosaic symptoms. 
In Nicotiana glutinosa the cowpea and sann-hemp viruses both behave similarly, but in their different forms produce very different lesions. The tobacco forms behave like type TMV. Under ordinary glasshouse conditions, local lesions appear in about $48 \mathrm{hr}$. and gradually increase in size. Their size and character varies with the age of the leaf inoculated and with the state of growth of the plants and all those on one leaf are not uniform, but in their most usual form they reach a diameter of 3-4 $\mathrm{mm}$. and are clearly defined with a light centre surrounded by a ring of dark-brown or black (Pl. 1, fig. 3). The lesions produced by the bean forms of the viruses do not become visible until about 4 days after inoculation and they are much smaller and less well defined; they also vary with the age and condition of the plant, but few reach a diameter greater than $1 \mathrm{~mm}$., and although some have partial rings of black, most are simply white flecks (Pl. 1, fig. 3). The differences between the lesions are large enough easily to distinguish the presence of small amounts of the tobacco forms in the presence of much of the bean forms.

In Nicotiana glutinosa also the results of infection depend on the temperature at which the inoculated plants are kept. At usual temperatures infection by both forms is restricted to the local lesions. At $36^{\circ}$ the tobacco form, like type TMV, causes large chlorotic local lesions (Pl. 1, fig. 4) and a systemic mottling; when plants with these symptoms are placed at $20^{\circ}$, the chlorotic areas soon become necrotic. At $30-36^{\circ}$ the bean form multiplies in inoculated $N$. glutinosa leaves and reaches greater amounts than at $20^{\circ}$ but, as in tobacco, causes no symptoms (Pl. 1, fig. 4); the areas in which the virus multiplies become necrotic soon after plants are placed at $20^{\circ}$.

In the necrotic lesions formed on Nicotiana glutinosa kept and infected under normal glasshouse conditions, the tobacco forms of both the cowpea and the sann-hemp viruses seem to multiply more extensively than the bean forms, for when single lesions are cut out, macerated and used to inoculate other $N$. glutinosa, many infections are obtained from lesions caused by the tobacco forms and few from those caused by the bean forms of the virus. However, this conclusion is not necessarily correct, because weight for weight the tobacco forms of the viruses are considerably more infective towards $N$. glutinosa than are the bean forms. To quote one comparison with purified preparations of the cowpea virus, in which opposite half leaves were rubbed with the two forms at concentrations of $100,10,1$ and $0.1 \mathrm{mg} . / 1$, the bean form produced average numbers of lesions/half leaf of 35, 16, 4 and $0 \cdot 1$ and the tobacco form produced $60,34,12$ and 5 . That this difference was not produced by any extra inactivation of the bean form during the processes of purification was shown by tests with clarified sap, when again sap from systemically-infected tobacco plants was ten or more times as infective per unit of virus as measured serologically as sap from systemically-infected beans. The virus content of sap from both tobacco and bean leaves varies with the condition of the plants and the time they have been infected, but tobacco sap usually contains 5-10 times more than bean sap. Hence, in comparisons with sap at different dilutions, similar numbers of 
lesions are produced by the two when tobacco sap is diluted about 100 times more than bean sap.

From single lesions produced in Nicotiana glutinosa, ten separate cultures of the cowpea virus were established, six produced by virus coming from tobacco and four from bean. Each culture was transferred serially four times in $N$. glutinosa and each time the inoculum for the next $N$. glutinosa was derived from one local lesion well separated from any other. The type of lesion remained constant during these transfers, the virus originating from bean producing each time only a few, small, slowly developing lesions and that originating from tobacco producing many more, larger and rapidly developing ones. After the fourth such transfer, sap from $N$. glutinosa infected from single lesions was used to inoculate bean and tobacco plants. Of the 6 cultures derived from the tobacco form, all immediately infected tobacco systemically and 2 bean; the 4 that did not immediately infect bean did so when inoculations were later made from the tobacco plants infected with them. Similarly, of the 4 cultures derived from the bean form, all infected bean directly from $N$. glutinosa and only 1 gave a systemic infection in tobacco, but subsequent inoculations from all the infected beans to tobacco gave systemic infections. The subsequent behaviour of none of these single-lesion cultures differed from that of the original bulk culture, and each when transferred from bean to tobacco underwent comparable changes in properties.

\section{Serological behaviour}

Rabbits were bled 7 days after being injected separately with $10 \mathrm{mg}$. of each of the forms of the cowpea virus. Both antisera precipitated with their homologous antigens, but only at dilutions up to $1 / 40$; the antiserum to the bean form also precipitated the heterologous antigen, but the antiserum to the tobacco form did not. When bled 7 days after a third injection of $10 \mathrm{mg}$., both antisera had precipitation titres greater than 1/1000 and both now precipitated their homologous and heterologous antigens. Their precipitation behaviour with the two forms, however, differed considerably. Not only was the precipitation titre of the sera much smaller with the heterologous than with the homologous antigens, but, as Table 1 shows, in tests with decreasing antigen and constant antiserum concentrations the homologous antigens precipitated more rapidly and over a much wider range of antigen/antibody ratios than did the heterologous antigens. When either antiserum was fully absorbed by the heterologous antigen, its ability to precipitate the homologous antigen was only slightly impaired. These facts combine to show that the two forms of the virus have very few antigens in common, and to suggest differences between the two of the same order as those between type TMV and cucumber virus 3 (Bawden \& Pirie, 1937), which has a known host range confined to the cucurbitaceae and which Knight (1955) thought so different from type TMV that, despite some common antigens, it should be regarded as a distinct virus.

Precipitation tests were also made with the two antisera and other strains of tobacco mosaic virus. The sann-hemp virus behaved very similarly to the cowpea virus, and although its two forms also probably have fewer than $10 \%$ 
Table 1. The precipitation of the bean and tobacco forms of the cowpea virus by their homologous and heterologous antisera

Times are the intervals between the beginning of incubation at $50^{\circ}$, after mixing antigen and antibody, and the recording of the amount of precipitation.

Bean form antigen at mg./l.

\begin{tabular}{|c|c|c|c|c|c|c|c|}
\hline & & 100 & & 25 & 12 & 6 & 3 \\
\hline Antiserum and & & \multicolumn{6}{|c|}{ Degree of precipitation } \\
\hline Bean, $1 / 20$ & $\begin{array}{l}5 \mathrm{~min} . \\
4 \mathrm{hr} .\end{array}$ & $\begin{array}{c}+++ \\
++++\end{array}$ & $\begin{array}{c}++ \\
+++\end{array}$ & $\begin{array}{c}t \\
++\end{array}$ & - & $\bar{t}$ & $\begin{array}{l}- \\
\pm\end{array}$ \\
\hline Bean, 1/100 & $\begin{array}{l}5 \text { min. } \\
4 \mathrm{hr} \text {. }\end{array}$ & $\begin{array}{l}+t+ \\
+t+\end{array}$ & $\begin{array}{c}++ \\
+++\end{array}$ & $\stackrel{+}{++}$ & $\overline{t+}$ & + & $\overline{+}$ \\
\hline Tobacco, $1 / 20$ & $\begin{array}{l}30 \mathrm{~min} \text {. } \\
4 \mathrm{hr} \text {. }\end{array}$ & - & $\begin{array}{c}+ \\
++\end{array}$ & $\stackrel{+}{+}$ & $\bar{t}$ & $\overline{ \pm}$ & - \\
\hline \multirow[t]{4}{*}{ Tobacco, 1/100 } & $\begin{array}{l}2 \mathrm{hr} . \\
4 \mathrm{hr} .\end{array}$ & - & - & $\overline{ \pm}$ & $\begin{array}{l}+ \\
+\end{array}$ & $\overline{ \pm}$ & - \\
\hline & & \multicolumn{6}{|c|}{ Tobacco form antigen at $\mathrm{mg} . / \mathrm{l}$. } \\
\hline & & 100 & 50 & 25 & 12 & 6 & $\mathbf{3}$ \\
\hline & & \multicolumn{6}{|c|}{ Degree of precipitation } \\
\hline Bean, 1/20 & $\begin{array}{l}30 \mathrm{~min} . \\
4 \mathrm{hr} .\end{array}$ & $\bar{t}+$ & $\begin{array}{c}+ \\
++\end{array}$ & $\overline{+}+$ & $\bar{t}+$ & - & 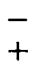 \\
\hline Bean, 1/100 & $4 \mathrm{hr}$. & - & - & - & - & + & + \\
\hline Tobacco, 1/20 & $\begin{array}{l}5 \mathrm{~min} . \\
4 \mathrm{hr} .\end{array}$ & $\begin{array}{c}+++ \\
++++\end{array}$ & $\begin{array}{c}++ \\
++++\end{array}$ & $\stackrel{+}{++t}$ & $\bar{t}+$ & + & $\overline{+}$ \\
\hline Tobacco, 1/100 & $\begin{array}{l}5 \mathrm{~min} . \\
4 \mathrm{hr} .\end{array}$ & $\begin{array}{c}+++ \\
++++\end{array}$ & $\begin{array}{c}++ \\
+++\end{array}$ & $\stackrel{+}{+++}$ & $\bar{t}+$ & - & $\bar{t}$ \\
\hline
\end{tabular}

Signs $(++++,++,++,+, \pm)$ indicate the relative sizes of the precipitates; $-=$ no precipitate

of their antigens in common, each is serologically similar to the comparable form of the cowpea virus. The other strains tested all behaved with the antiserum against the tobacco form in much the same way as they do with antisera to type TMV, which obviously is antigenically very similar to the tobacco forms of the cowpea and sann-hemp viruses. When tested against the antiserum to the bean form, type TMV, tomato aucuba mosaic virus and the tobacco form of the sann-hemp virus were all precipitated to much the same extent as the tobacco form of the cowpea virus, but strain U2 (Siegel \& Wildman, 1954) and one that causes a systemic mosaic in Datura tatula were not precipitated. That these two strains probably have a very few antigens in common with the bean form, however, was suggested by the formation of small precipitates when a preparation of the bean form of the cowpea virus was titrated against their antisera; precipitates formed only when these antisera, which with their homologous antigens have titres in excess of 1/1000, were used at dilutions less than $1 / 25$, and then they formed over only a narrow zone of antigen/antibody ratios and after prolonged incubation at $50^{\circ}$. No 
attempt was made to assess the precise degree of serological relationships between the different strains by detailed cross-absorption experiments. The results, indeed, would be of doubtful value, for the antibody contents of different sera prepared against one preparation of one strain differ qualitatively as well as quantitatively, depending on the amount of antigen injected, the number of injections and the relative extent to which the animal responds to quantitatively major and minor antigens.

Other strains of tobacco mosaic virus that have been found to differ considerably in their antigenic constitution have been found also to have considerably different amino acid compositions. Analyses made by $\mathbf{M r} \mathbf{M}$. W. Rees of the Biochemical School, Cambridge University, show that there are also considerable differences between the amino acid compositions of the two forms of the cowpea virus. The most striking is that the bean form contains histidine, which does not occur in the tobacco form and has been previously recorded in only the rib-grass strain of TMV (Knight, 1947), but it also contains more arginine and less lysine than the tobacco form. That the histidine is not present in a normal bean protein contaminating the purified preparation of the bean form was shown by propagating this form of the virus in tobacco. This was done by inoculating tobacco plants with the bean form, keeping them at $32^{\circ}$ continuously for 8 days, when the inoculated leaves were harvested and the virus purified. The preparation caused only small slowly developing lesions when inoculated to Nicotiana glutinosa, precipitated with antisera in the manner of the bean form and Mr Rees found it to contain the same amount of histidine as preparations made from systemically-infected beans.

\section{Electrophoretic behaviour}

When purified preparations of the tobacco form were examined at $\mathbf{0 . 2} \%$ in the electrophoresis apparatus they behaved much like preparations of type TMV and showed a single component with a tall slim peak of mobility about $-9 \cdot 4 \times 10^{-5} \mathrm{~cm}^{2} \mathrm{sec}^{-1} \mathrm{~V} .^{-1}$ (Pl. 2, fig. 5), but at $20 \mathrm{mg}$. $/ \mathrm{ml}$. another component with mobility $-4.7 \times 10^{-5} \mathrm{~cm}^{2} \mathrm{sec}^{-1} \mathrm{~V} .^{-1}$ was detectable. Preparations of the bean form at $2 \mathrm{mg}$. $/ \mathrm{ml}$. usually showed two distinct components (Pl. 2, fig. 6), the major with a mobility of about -4.7 and the minor of $-9 \cdot 4 \times 10^{-5} \mathrm{~cm} .^{2}$ sec. ${ }^{-1}$ V..$^{-1}$. Hence, components with the same mobilities are detectable in preparations of both forms of the virus, but the component that is minor in one becomes the major in the other.

At $20 \mathrm{mg} . / \mathrm{ml}$. there was evidence of additional components in the bean form, and as shown below, these were also once noted at lower concentrations. The peaks are less slim than with the tobacco form and broaden more as electrophoresis proceeds. This suggests that the particles are smaller, as also does the fact that the bean form precipitates with its antiserum more slowly and its floccules are more compact than those of the tobacco form. Electron micrographs further suggest this, for those of purified preparations of the bean form show most particles to be shorter than $300 \mathrm{~m} \mu$., unlike preparations of the tobacco form, but no attempt was made to estimate the difference by counting random particles of the two. Also, ultracentrifugation adequate to sediment 
preparations of the tobacco form leaves material in the supernatant fluid of purified preparations of the bean form. This material was not studied in any detail but electron micrographs of the supernatant fluid after centrifuging for $1 \mathrm{hr}$. at $50,000 \mathrm{~g}$ showed it to contain few particles as long as $100 \mathrm{~m} \mu$. and, weight for weight, it had only $\frac{1}{50}$ th the infectivity of the material that sedimented. It absorbed ultraviolet radiation at $2600 \AA$ rather less strongly than normal preparations, suggesting that it probably contained less than $6 \%$ nucleic acid. When examined electrophoretically at about $2 \mathrm{mg} . / \mathrm{ml}$. it gave a single peak moving with the speed of the minor component of the preparations of the bean form (Pl. 1, fig. 7). However, centrifugation did not separate the two components completely, for solutions of the re-suspended pellet contained both.

There are obvious similarities between the small particles present in the preparations of the bean form of the cowpea virus and the material that Takahashi \& Ishii (1953) called X-protein, which remains in the supernatant fluid when sap from tobacco plants infected with type TMV is ultracentrifuged. However, free $\mathrm{X}$-protein does not accompany TMV through purification procedures and it forms a much smaller proportion of the total anomalous protein present in sap than does the minor component of the bean-form preparations. The ratio of the minor and major components varied considerably in different preparations of the bean form of the cowpea virus, but whether this reflects variations in the amounts originally present in the sap or variable losses of the minor component during purification is unknown. Every preparation from bean was found to have at least these two components, but when the purified preparation of the bean form from inoculated tobacco plants kept at $32^{\circ}$ was examined at $2 \mathrm{mg} . / \mathrm{ml}$., this showed only one, which had the mobility of the major component of the bean preparation. Again, it is unknown whether this was because the minor component is not formed under these conditions or whether it was lost during purification.

That the minor component in preparations of the bean form contains infective particles was shown by separating the two components electrophoretically. When inoculated to Nicotiana glutinosa at similar concentrations each gave similar numbers of the small slowly developing lesions. Each also produced similar systemic symptoms on French beans as well as many local lesions of the type shown in Pl. 1, fig. 1. When virus prepared from beans inoculated with either ;the separated major or minor component was examined at $2 \mathrm{mg} . / \mathrm{ml}$., it gave electrophoretic patterns with the usual two components. Tobacco plants inoculated with either the minor or major component all produced local lesions; not all became systemically infected and more did from inoculations with the separated minor component than from those with the major. Later inoculations from beans infected with either of the two separated components produced systemic infections in tobacco regardless of whether the component had previously infected tobacco systemically.

Serological tests with the two separated components showed no great differences between their behaviour. Each was precipitated by antiserum against the bean and tobacco forms of the cowpea virus, and each was precipitated more rapidly and over a wider range of antigen/antibody ratios by 
the bean-form antiserum than by the tobacco-form antiserum. Thus although the minor component has much the same electrophoretic mobility as the tobacco form of the virus, it seems to be no more similar to it antigenically than is the major component, which moves much more slowly.

The sann-hemp virus behaved in much the same way as the cowpea one. Purified preparations of the tobacco form at $2 \mathrm{mg} . / \mathrm{ml}$. behaved like type TMV and showcd one component; preparations of the bean form showed a major component with mobility -4.2 and a minor one with mobility $-9.7 \times 10^{-5} \mathrm{~cm}^{2} \mathrm{sec}^{-1} \mathrm{~V} .{ }^{-1}$. When these two components were separated electrophoretically and inoculated to Nicotiana glutinosa, each produced only small slowly developing local lesions. Each infected beans systemically, produced local lesions in tobacco and infected some plants systemically. All purified virus preparations from beans infected systemically by inoculation with either component again showed the two usual components when examined electrophoretically, and the preparation from one batch of beans inoculated with a separated minor component showed two additional components with approximate mobilities of -7 and $-5 \times 10^{-5} \mathrm{~cm} \cdot .^{2} \mathrm{sec} .^{-1} \mathrm{~V} .^{-1}$ (Pl. 2, fig. 8).

\section{Inactivation by ultraviolet radiation}

Table 2 shows the results of infection tests with unirradiated preparations of the bean and tobacco forms of the cowpea virus and with samples of the same preparations after exposure for different lengths of time to ultraviolet

\section{Table 2. The inactivation of the bean and tobacco forms of the coropea virus} by ultraviolet radiation

Preparations at $200 \mathrm{mg} . / \mathrm{l}$. were irradiated as described in the text and diluted to the stated concentrations before inoculation to Nicotiana glutinosa.

Treatment

Unirradiated
Unirradiated
Unirradiated
Irradiated, 40 sec.
Irradiated, 80 sec.
Irradiated, 120 sec.

Unirradiated
Unirradiated
Unirradiated
Irradiated, 80 sec.
Irradiated, 160 sec.
Irradiated, 240 sec.

$\begin{array}{cc}\begin{array}{c}\text { Inoculum } \\ \text { concentration (mg./l.) }\end{array} & \begin{array}{c}\text { Mean no. lesions/ } \\ \text { half leaf }\end{array} \\ \text { Bean form } & \\ 10 & 36 \\ 3.3 & 25 \\ 1 \cdot 0 & 8 \\ 20 & 4.5 \\ 66 & 3.5 \\ 200 & 2 \cdot 3\end{array}$

Tobacco form

$\begin{array}{cc}10 & 98 \\ 3 \cdot 3 & 43 \\ 1 \cdot 0 & 30 \\ 20 & 65 \\ 66 & 26 \\ 200 & 13\end{array}$

(u.v.) radiation. The source of the radiation was a Vitreosil low-pressure mercury lamp, most of whose radiation is of wavelength $2537 \AA$. Solutions containing $200 \mathrm{mg}$. purified virus preparation $/ 1$. were irradiated as layers $0 \cdot 14 \mathrm{~cm}$. deep in Petri dishes placed $20 \mathrm{~cm}$. from the lamp, where the intensity of the radiation was $870 \mu W . / \mathrm{cm} .^{2}$. The dishes were rocked continually during the period 
of exposure, a treatment that is assumed to have ensured that all particles were equally exposed. The control and irradiated preparations were then inoculated at various dilutions to Nicotiana glutinosa. The tobacco form is obviously much more resistant to inactivation than the bean form; after exposure for $160 \mathrm{sec}$. it still had about $\frac{1}{60}$ th of its initial infectivity, whereas the bean form retained only about $\frac{1}{40}$ th after 40 sec. and less than $\frac{1}{500}$ th after exposure for 120 sec. Siegel \& Wildman (1954) found that different strains of tobacco mosaic virus differed in their sensitivity to inactivation by u.v. radiation and the differences between the two forms of the cowpea virus seem to be of the same order as those between the resistant type TMV and their most sensitive strain U2. Strain U2, which was derived from a stock of type TMV, also has the same electrophoretic mobility as the major component in bean preparations of the cowpea and sann-hemp viruses, but it seems to be genetically more stable than the cowpea virus, for Siegel \& Wildman (1954) stated that it 'never reverted to common TMV in spite of numerous transfers over a 3-year period'. It also gives smaller local lesions in $N$. glutinosa than does type TMV, but these appear at the same rate; I could get no infections in French beans with strain U2, which despite some similarities with the bean form of the cowpea virus also seems to have fewer antigens in common with it than does type TMV.

The change from bean to tobacco form

When the tobacco form of the cowpea virus is mixed in various amounts with a constant amount of the bean form and the mixtures inoculated to Nicotiana glutinosa, the lesions produced by the tobacco form appear in the usual time of 2 days and they remain readily distinguishable from those produced later by the bean form. As the number of lesions produced by the tobacco form also seems little affected by the presence of the bean form, inoculations to $N$. glutinosa provide a convenient method of detecting when the tobacco form first appears in leaves inoculated with the bean form. This change-over was studied by inoculating leaves with the bean form, washing them thoroughly to get rid of virus on the leaf surface, and then macerating samples from them at intervals for inoculation to $N$. glutinosa.

When tobacco leaves are inoculated with the tobacco form of the cowpea virus less virus is usually recovered in extracts $24 \mathrm{hr}$. after inoculation than $1 \mathrm{hr}$. afterwards, but after $48 \mathrm{hr}$. the amount has usually increased considerably; after 3 days there is usually detectable multiplication, even when the original inoculum was highly diluted, and from then on the amount increases rapidly. When similar leaves are inoculated with undiluted infective bean sap, or better (because bean sap contains substances that interfere with infections in tobacco and Nicotiana glutinosa) with $1 \mathrm{mg} . / \mathrm{ml}$. of purified beanform virus, extracts made 24 or $48 \mathrm{hr}$. after inoculation usually contain less virus than extracts made $1 \mathrm{hr}$. after inoculation. Newly produced virus begins to be detectable about 3-4 days after inoculation, when the leaves start to show lesions, but the number of lesions produced on $N$. glutinosa by extracts taken at this time are few compared with those from plants inoculated with the 
tobacco form. All the lesions at this time are of the small slowly developing type, and the number of such lesions does not increase greatly with increase in the period of time at $20^{\circ}$ between inoculation of the original tobacco leaves and the preparation of the extracts. From many inoculated leaves, this is the only result of such return inoculations to $N$. glutinosa, but extracts from leaves of plants that will later develop systemic mosaic symptoms sooner or later give, in addition, the large rapidly developing lesions which are characteristic of infection by the tobacco form of the virus (Pl. 2, fig. 9). These large lesions rarely occur from inoculations with extracts made sooner than 6 days after the tobacco leaves were inoculated and sometimes not until twice as long. Extracts made at intervals after the first appearance of the large lesions usually produce large lesions in rapidly increasing numbers, but not always so, presumably because sometimes the first sampling of the tobacco leaves has removed the only site or sites where the tobacco form of the virus was multiplying.

No exhaustive search has been made to find the range of plants in which the change from bean to tobacco form occurs, but it is not restricted to tobacco. It occurs in other species of Nicotiana, such as $N$. bigelovii and $N$. clevelandii, but has not been noticed in $N$. glutinosa; it also occurs in other solanaceous plants, such as Datura tatula and the tomato, and in at least one plant from the Chenopodiaceae, namely sugar beet. From leaves of these species inoculated with preparations of the bean form, which themselves gave no large rapidly developing lesions in $N$. glutinosa, extracts made during the first few days after inoculation also produced only lesions characteristic of infection with the bean form, whereas extracts made after a week or more produced lesions of both types.

Studies of the course of infection when bean leaves are inoculated with the tobacco form of the cowpea virus are complicated by the small virus content of such leaves and the presence of substances that inhibit infections in Nicotiana glutinosa. However, such tests as were made showed that virus obtained in extracts made within 5 days of inoculation produced only lesions characteristic of infection by the tobacco form, whereas later ones often produced a few lesions characteristic of infection by the bean form.

\section{DISCUSSION}

The main point that calls for discussion is the mechanism whereby the cowpea and sann-hemp viruses change their constitution and properties when they are transferred between solanaceous and leguminous hosts. The novelty about these viruses is the magnitude of the changes they undergo and that the changes are reversible, for there is nothing new in the discovery that strains of TMV vary or that the behaviour of cultures alters when they are passed through a new host. Most variations that have previously been described, however, have been changes in virulence, although strain U2 which seems to have derived from type TMV also differs from it in several other ways (Siegel \& Wildman, 1954). Also, changes resulting from passage through a new host plant have previously been explicable by inocula containing more than one 
strain, not all of which invade the new host systemically. Thus Johnson (1947), who found that bulk cultures of TMV were always less virulent towards tobacco after passage through sea-holly (Eryngium aquaticum L.), readily explained this by experiments with single-lesion isolates, which showed that bulk cultures contained both virulent and avirulent forms and that the virulent ones gave only local lesions in sea-holly. Single-lesion isolates of the cowpea virus, however, changed as readily as did bulk cultures, and the reversibility of the change also seems inexplicable by postulating that different hosts act solely by differentially favouring one strain out of several introduced in the inoculum.

It cannot safely be assumed that individual lesions in Nicotiana glutinosa are caused by single virus particles, but what evidence there is suggests that it is rare for widely separated lesions, produced by dilute inocula in which two strains are mixed, to contain both strains (Kunkel, 1934; Lauffer \& Price, 1945). Although the 'purity' of any culture is always suspect, it seems improbable that the tobacco form of the cowpea virus is carried as such through local lesions produced by inoculations with the bean form. Not only does the tobacco form produce its characteristic lesions when it is added in small amounts to large quantities of the bean form, but it produces them in approximately the same numbers as when inoculated on its own. Also, even if its presence were not immediately obvious from the character of the lesion, it should become so after a second passage in $N$. glutinosa, for extracts from lesions caused by the tobacco form are much more infective than those from the bean form. However, when the single-lesion isolates were passed successively through $N$. glutinosa, they gave no sign of containing the tobacco form.

Similarly, the results of inoculating Pinto beans give no reason to suggest that sap from systemically-infected tobacco contains both forms of the virus in transmissible amounts. This variety gives only black local lesions when inoculated with the tobacco form of the virus and no such local lesions but a systemic mosaic disease when inoculated with the bean form. Inoculations from such mottled leaves can cause systemic infections in tobacco, but the virus that becomes systemic gives only black local lesions when returned to Pinto. When inoculated to Prince instead of Pinto beans, it can lead to a systemic infection, and the virus from Prince will then readily infect Pinto systemically and cause no black local lesions. Thus, starting with virus that is causing a systemic mosaic disease in Pinto, the ability to do this is lost by passage through tobacco and regained by passage from tobacco through Prince.

None of these results seems compatible with the idea that hosts act as one-way filters, which allow only one of the types that initially infect to pass the inoculated leaves. Nor does the fact that inoculating tobacco plants with the bean form of virus leads to the production of the tobacco form in young plants at $20^{\circ}$, but not at $30^{\circ}$ or in older plants, for when present the tobacco form multiplies readily in all three types of plant. Such facts and many others all combine to suggest that the tobacco form occurs in tobacco plants inoculated with the bean form, not because of its presence in the inoculum, but only after the bean form has multiplied there and because of this multiplication. 
The change from either form of the virus to the other seems explicable only by the hosts selecting variants that originate in them. An attempt was made (Bawden, 1956) to explain the differences between the two forms as phenotypic variations, with the genetical material of the virus remaining constant but combining with different proteins in solanaceous and leguminous hosts. This now seems improbable, for since Gierer \& Schramm (1956) showed that nucleic acid of TMV can alone cause infections, the idea has become to some extent testable. When preparations of the cowpea virus in its two forms were disrupted with phenol to produce infective preparations of nucleic acid, each form still produced its characteristic type of local lesion in Nicotiana glutinosa.

The only objection to the idea that the two forms can change into one another by events equivalent to reversible mutations seems to rest on the apparent suddenness with which such large changes occur. The differences between the two forms are perhaps greater than those between any two previously recognized strains of TMV, and most other strains that differ considerably can reasonably be assumed to have reached their current states by changes accumulated over long periods of evolution along separate paths. However, differences in the size and rate of change seems no adequate reason for postulating a different mechanism. In terms of population statistics, there is no reason to assume that the change happens so very often or that it happens as a 'single-step' mutation. With the best conditions for the change from the bean to the tobacco form, not every tobacco plant becomes systemically infected, even when 3-4 leaves are dusted with 'Celite' and rubbed over their whole surfaces with inoculum of the bean form at $1 \mathrm{mg} . / \mathrm{ml}$. Such a treatment may give hundreds of local infections per leaf and each lesion will contain many millions of virus particles, produced by multiplication cycles in a succession of infected cells. Possibly only one particle of the tobacco form needs to occur in such conditions to lead to a systemic infection, so the mutation rate need not be fantastically high. Furthermore, if the many differences in behaviour between the two forms reflect many genetical differences, not all the required mutations need necessarily have occurred in one particle; in the course of multiplication, particles in which different mutations occur may be able to exchange genetic material.

The multiplication of type TMV does not lead to a uniform product all exactly resembling the infecting particles, but to a variety of products differing in constitution, size and infectivity (Bawden \& Pirie, 1945, 1956). That there is even a greater variability in the products when the cowpea virus multiplies, especially in beans, is evident from the electrophoretic patterns. Infectivity tests give no evidence that the variability extends to the occurrence in sap from beans of particles with the infective characters of the tobacco form, for the typical lesions in Nicotiana glutinosa were not formed even when inoculations were made with the component having the same electrophoretic mobility as the tobacco form. However, this failure does not mean that no such particles occur. To get infections by rubbing leaves requires the use of inocula containing at least $10^{7}$ particles/ml., so that many could lie hidden in sap from beans. Hence, when multiplying in any host each form of the virus 
may occasionally produce particles of the other form, but only when the occasional particles are produced in hosts which they, but not their parents, readily invade systemically can they multiply and reach concentrations at which their presence becomes detectable. The only experimental evidence that seems difficult to reconcile with this idea of undirected and random mutations leading from one form to another, is the failure of large tobacco plants to become systemically infected by inocula of the bean form that give systemic infections in young seedlings, for the tobacco form seems to multiply and move with almost equal readiness in plants of both ages. One explanation might be that the bean form itself multiplies much less in the larger plants so that, with a constant mutation rate, there would be fewer opportunities for the tobacco form to be produced. However, the possibility of the species and physiological condition of the host plant playing some part in producing mutations, in addition to selecting those forms best able to invade it, cannot be excluded.

That variants do occur in single-lesion isolates of the tobacco form when multiplying in tobacco is easily demonstrable by selecting inoculum from the occasional yellow spots that occur. When this was used to infect further tobacco plants, it produced a general yellow mosaic; inoculations from such plants infected Prince beans systemically, but produced the same kind of symptoms as did unselected inoculum. Also, when returned to tobacco, the systemic symptoms there were again of the usual type; similarly, when continually passaged in tobacco without selecting inoculum from the yellow areas, the symptoms reverted to the original type. Hence, it seems that the reversible changes can be of more than one type, some simply affecting virulence towards tobacco without greatly altering the physico-chemical properties, and others leading to much larger differences.

In addition to those mentioned in the text, I am also greatly indebted to Dr A. Kleczkowski for making all the electrophoretic measurements and separations and to Mr H. L. Nixon for making electron micrographs.

\section{REFERENCES}

Bawden, F. C. (1956). Reversible, host-induced, changes in a strain of tobacco mosaic virus. Nature, Lond. 177, 302.

Bawden, F. C. \& PIRIE, N. W. (1937). The relationship between liquid crystalline preparations of cucumber viruses 3 and 4 and strains of tobacco mosaic virus. Brit. J. exp. Path. 18, 275.

Bawden, F. C. \& Pirie, N. W. (1940). The effects of alkali and some simple organic substances on three plant viruses. Biochem. J. 34, 1278.

Bawden, F. C. \& Pirie, N. W. (1945). The separation and properties of tobacco mosaic virus in different states of aggregation. Brit. J. exp. Path. 26, 294.

Bawden, F. C. \& Pirie, N. W. (1956). Observations on the anomalous proteins occurring in extracts from plants infected with strains of tobacco mosaic virus. J. gen. Microbiol. 14, 460 .

Capoor, S. P. (1950). A mosaic disease of sann hemp in Bombay. Curr. Sci. 19, 22.

Grerer, A. \& Schramm, G. (1956). Infectivity of ribonucleic acid from tobacco mosaic virus. Nature, Lond. 177, 702.

JoHnson, J. (1947). Virus attenuation and the separation of strains by specific hosts. Phytopathology, 37, 822. 
KNIGHT, C. A. (1947). The nature of some of the chemical differences among strains of tobacco mosaic virus. J. biol. Chem. 171, 297.

KNIGHT, C. A. (1955). Are cucumber viruses 3 and 4 strains of tobacco mosaic virus? Virology, 1, 261.

KUNKEL, L. O. (1934). Tobacco and aucuba-mosaic infections by single units of virus. Phytopathology, 24, 13 (Abstr.).

Lauffer, M. A. \& Price, W. C. (1945). Infection by viruses. Arch. Biochem. 8, 449.

Lister, R. M. \& Thresh, J. M. (1955). A mosaic disease of leguminous plants caused by a strain of tobacco mosaic virus. Nature, Lond. 175, 1047.

Price, W. C. (1930). Local lesions on bean leaves inoculated with tobacco mosaic virus. Amer. J. Bot. 17, 694.

Stegel, A. \& Wildman, S. G. (1954). Some natural relationships among strains of tobacco mosaic virus. Phytopathology, 44, 277 .

Silberschmidt, K. \& Kramer, M. (1941). Brazilian bean varieties as plant indicators for the tobacco-mosaic virus. Phytopathology, 31, 430.

Takahashi, W. N. \& Ishir, M. (1953). A macromolecular protein associated with tobacco mosaic virus infection: its isolation and properties. Amer. J. Bot. 40, 85.

\section{EXPLANATION OF PLATES}

\section{Prate 1}

Fig. 1. A first-formed leaf of French bean (Phaseolus vulgaris) var. Prince, photographed 10 days after it was inoculated with the bean form of the cowpea virus, when it showed many yellow and white rings and spots.

Fig. 2. Deformed and mottled leaves from French bean, var. Prince, photographed 5 weeks after the plant was infected with the cowpea virus.

Fig. 3. Leaf of Nicotiana glutinosa showing the kinds of lesions produced by the bean and tobacco forms of the cowpea virus in plants kept under the usual glasshouse conditions. Photographed 1 week after inoculation: left-hand half of leaf shows the small white lesions produced by the bean form and the right-hand the larger lesions produced by the tobacco form.

Fig. 4. Leaf of Nicotiana glutinosa showing the large chlorotic rings (left-hand half) produced by the tobacco form of the cowpea virus in plants kept continuously at $36^{\circ}$; the right-hand half of the leaf is infected with the bean form, which multiplies at this temperature but causes no lesions. Photographed 1 week after leaf was inoculated.

\section{Prate 2}

Fig. 5. Electrophoretic pattern of a purified preparation of the tobacco form of the cowpea virus, showing a single sharp peak with a mobility of $-9 \cdot 4 \times 10^{-5} \mathrm{~cm}^{2} \mathrm{sec}^{-1} \mathrm{~V} .{ }^{-1}$.

Fig. 6. Electrophoretic pattern of a purified preparation of the bean form of the cowpea virus showing two peaks, of which the larger has a mobility of -4.7 and the smaller of $-9 \cdot 4 \times 10^{-5} \mathrm{~cm}^{2}$ sec. $^{-1} \mathrm{~V} .^{-1}$.

Fig. 7. Electrophoretic pattern of a preparation, made from the unsedimented material after a purified preparation of the bean form of the cowpea virus was ultracentrifuged at $50,000 \mathrm{~g}$, showing a single peak with a mobility of $-9 \cdot 4 \times 10^{-5} \mathrm{~cm}^{2} \mathrm{sec}^{-1} \mathrm{~V} .{ }^{-1}$.

Fig. 8. Electrophoretic pattern of a purified preparation of the bean form of the sann-hemp virus, showing four components with mobilities of $-9 \cdot 4,-7,-5$ and $-4.7 \times 10^{-5} \mathrm{~cm}^{2}$ sec. ${ }^{-1} \mathrm{~V}^{-1}$. The French beans from which this preparation was made were infected by inoculation with the slower component, separated electrophoretically from a preparation that contained only the usual two.

Fig. 9. Leaf of Nicotiana glutinosa showing lesions produced by inoculation with extracts made from tobacco leaves one week after they were inoculated with the bean form of the cowpea virus. Right-hand half inoculated with 'Celite' to increase numbers of lesions. Most of the lesions are typical of those produced by the bean form, and this was the only type given by earlier extracts; but now there are also some larger and more distinctive lesions, typical of those produced by the tobacco form. 
Journal of General Microbiology, Vol. 18, No. 3
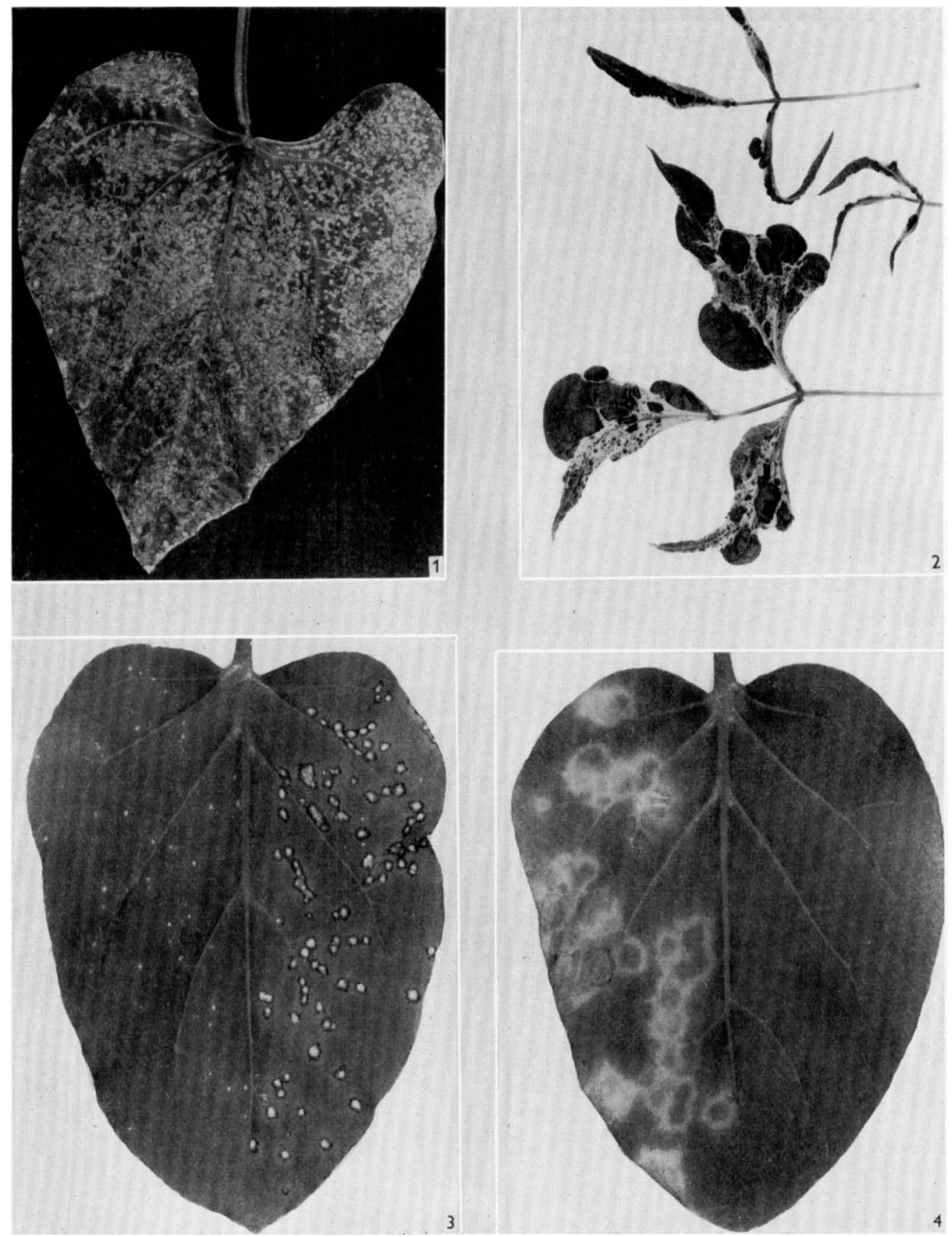

F. C. Bawdin-Reviersibi.f changes in tobacco mosaic viruses. Plate 1

(Facing $p$. 766) 
Journal of General Microbiology, Vol. 18, No. 3
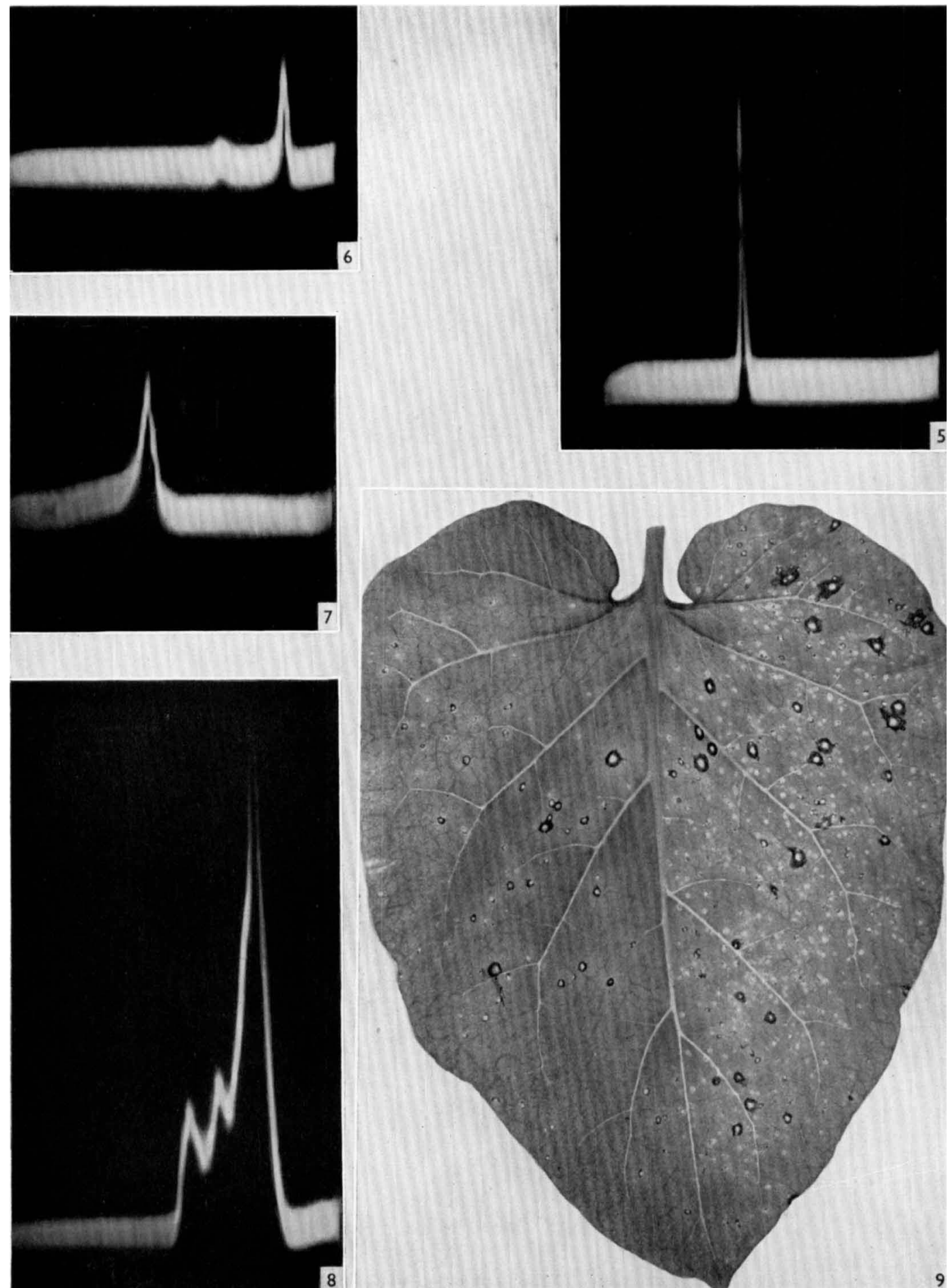

o

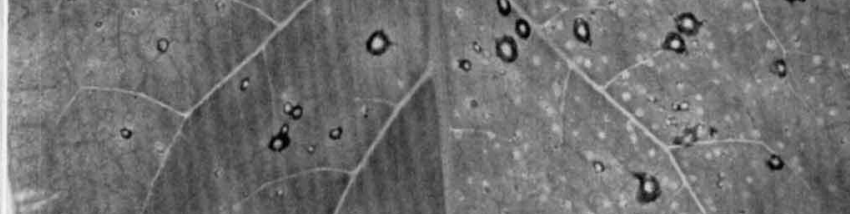

F. C. Bawden-Reversible changes in tobacco mosaic viruses. Plate 2 